\title{
Smouldering fire or conflagration? An illustrated update on the concept of inflammation in pulmonary arterial hypertension
}

\author{
Frédéric Perros ${ }^{1,2,3}$, Marc Humbert $\mathbb{1}^{1,2,4}$ and Peter Dorfmüller $\mathbb{C}^{5,6}$
}

${ }^{1}$ Université Paris-Saclay, School of Medicine, Le Kremlin Bicêtre, France. ${ }^{2}$ INSERM UMR S 999, Hôpital Marie Lannelongue, Le Plessis Robinson, France. ${ }^{3}$ Paris-Porto Pulmonary Hypertension Collaborative Laboratory (3PH), INSERM, Le Kremlin-Bicêtre, France. ${ }^{4}$ Assistance Publique - Hôpitaux de Paris (AP-HP), Dept of Respiratory and Intensive Care Medicine, Pulmonary Hypertension National Referral Center, Hôpital Bicêtre, Le Kremlin-Bicêtre, France. ${ }^{5}$ Institut für Pathologie, Universitätklinikum Giessen und Marburg, Giessen, Germany. ${ }^{6}$ Deutsches Zentrum für Lungenforschung (DZL), Giessen, Germany.

Corresponding author: Peter Dorfmüller (peter.dorfmuller@patho.med.uni-giessen.de)

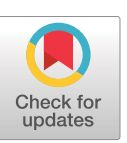

This version is distributed under the terms of the Creative Commons Attribution NonCommercial Licence 4.0. For commercial reproduction rights and permissions contact permissions@ersnet.org

Received: 7 July 2021 Accepted: 20 Oct 2021

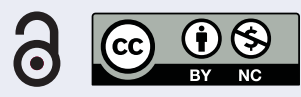

Shareable abstract (@ERSpublications)

Several pro-inflammatory triggers and pathways ignite and fuel persistent pulmonary vascular inflammation and obstructive vascular remodelling in PAH. Individual inflammatory susceptibility might reveal responder populations for immunomodulatory therapies. https://bit.ly/3GC1XMC

Cite this article as: Perros F, Humbert M, Dorfmüller P. Smouldering fire or conflagration? An illustrated update on the concept of inflammation in pulmonary arterial hypertension. Eur Respir Rev 2021; 30: 210161 [DOI: 10.1183/16000617.0161-2021].

\section{Abstract}

Pulmonary arterial hypertension (PAH) is a rare condition that is characterised by a progressive increase of pulmonary vascular resistances that leads to right ventricular failure and death, if untreated. The underlying narrowing of the pulmonary vasculature relies on several independent and interdependent biological pathways, such as genetic predisposition and epigenetic changes, imbalance of vasodilating and vasoconstrictive mediators, as well as dysimmunity and inflammation that will trigger endothelial dysfunction, smooth muscle cell proliferation, fibroblast activation and collagen deposition. Progressive constriction of the pulmonary vasculature, in turn, initiates and sustains hypertrophic and maladaptive myocardial remodelling of the right ventricle. In this review, we focus on the role of inflammation and dysimmunity in PAH which is generally accepted today, although existing PAH-specific medical therapies still lack targeted immune-modulating approaches.

\section{Introduction}

Pulmonary arterial hypertension (PAH) is a rare condition with an inevitable critical evolution and fatal outcome, if untreated. The disease is characterised by a progressive increase of pulmonary vascular resistance that eventually leads to right ventricular failure [1]. Diagnosis mostly relies on right heart catheterisation and exclusion of other aetiologies, such as thromboembolism, through imaging or ventilation/perfusion scan, since the remodelling process of lung vessels that causes an increase of pressure within the pulmonary circuit involves small peripheral vessels and microvessels that cannot be directly visualised by non-invasive techniques [1].

The pathophysiology of PAH is complex and has many pillars, such as genetic predisposition and epigenetic changes, an imbalance of vasodilating and vasoconstrictive mediators, as well as dysimmunity and inflammation that eventually lead to endothelial dysfunction, smooth muscle cell proliferation and fibroblast activation/collagen deposition within the thickening vessel walls. This in turn leads to hypertrophic and maladaptive myocardial remodelling of the right ventricle (figure 1) [2].

In this illustrated review, we will focus on the role of inflammation and dysimmunity, a generally accepted branch of the condition's pathophysiology, although existing PAH-specific medical therapies still lack targeted anti-inflammatory or immune-modulating approaches [3].

While the presence of perivascular inflammatory cells and auto-antibody generating lymphoid tertiary follicles in the vicinity of pulmonary arteries has been described in the past and the increase in 


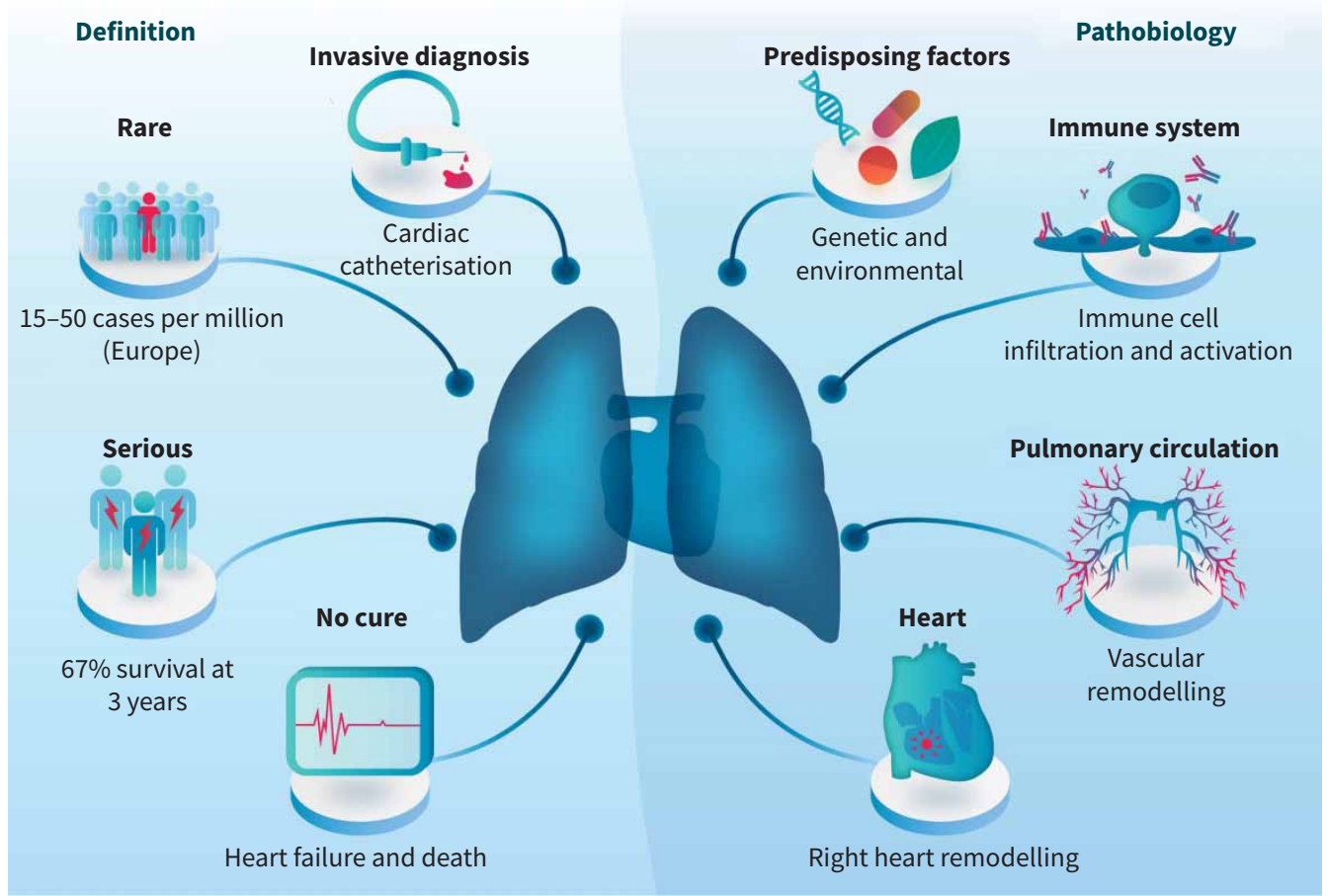

FIGURE 1 Pulmonary arterial hypertension characteristics.

pro-inflammatory cytokines and chemokines and auto-antibodies in the peripheral blood repeatedly reported, interpretations of the role, mechanistic rank and pathophysiologic relevance of these inflammatory and dysimmune hallmarks are still controversial [4]. This controversy is further fuelled by the fact that the most

High levels of cytokines and chemokines
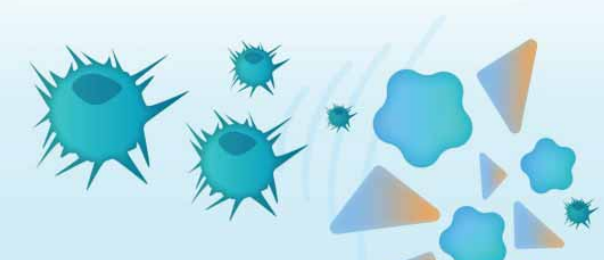

Inflammatory cells recruitment

Pulmonary vascular lesions
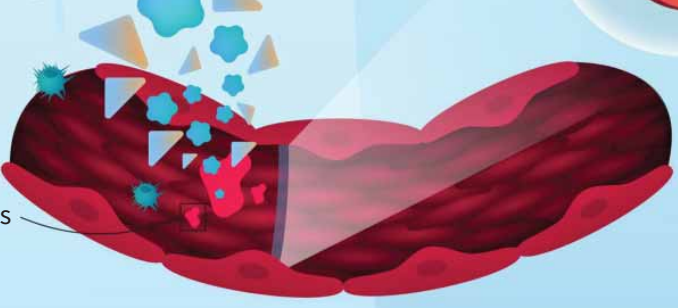

$10-40 \%$ of patients with idiopathic PAH

Associated with immune disorders
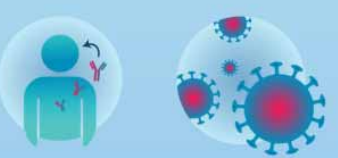

Main predisposing

genes involved

in immune regulation

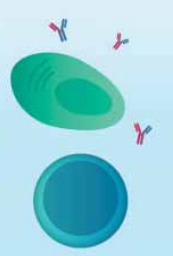

Auto-reactive $\mathrm{T}$ - and B-cells

Autoimmune diseases

HIV

FIGURE 2 Inflammatory mechanisms involved in pulmonary arterial hypertension (PAH) pathogenesis. 


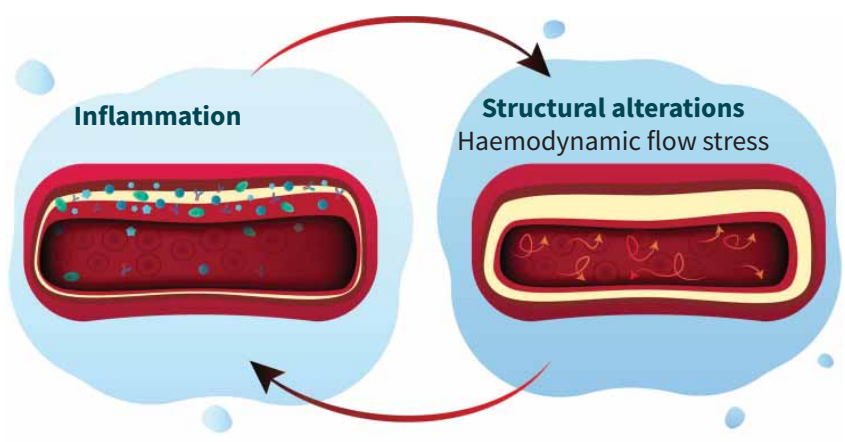

Which one is the follower or the driver?

FIGURE 3 The two types of mechanism that underlie pulmonary arterial hypertension pathogenesis.

prominent $\mathrm{PAH}$-associated mutations are critical for immune-regulatory processes, in addition to their relevance in vascular homeostasis and function [5]. It should be kept in mind, however, that PAH is frequently associated with primary autoimmune and immune disorders, such as connective tissue disease (CTD)-associated or HIV-associated PAH, where pulmonary hypertension (PH) develops in a clearly pro-inflammatory context (figure 2). For these conditions at least, increases in pulmonary arterial pressures can be positively influenced by anti-inflammatory and/or immune-modulatory therapy, a strategy that has not yet made its way to PAH-specific therapies in general, despite recent pharmacological trials [6].

One fundamental question remains still unanswered: why do obstructive lesions of small pulmonary arteries/arterioles/venules and perivascular chronic inflammatory infiltrate concur? Where does the story begin? Occlusion and reactive inflammation, or inflammation and reactive occlusion? The latter hypothesis has been a much-used rationale for many scientific reports in the past 30 years. The former option appears less inspiring, but should not be discarded too quickly, since the pathophysiologic importance of disease evolution can come from self-sustaining, smouldering and long-standing secondary fires, not only from initial conflagrations (figure 3).

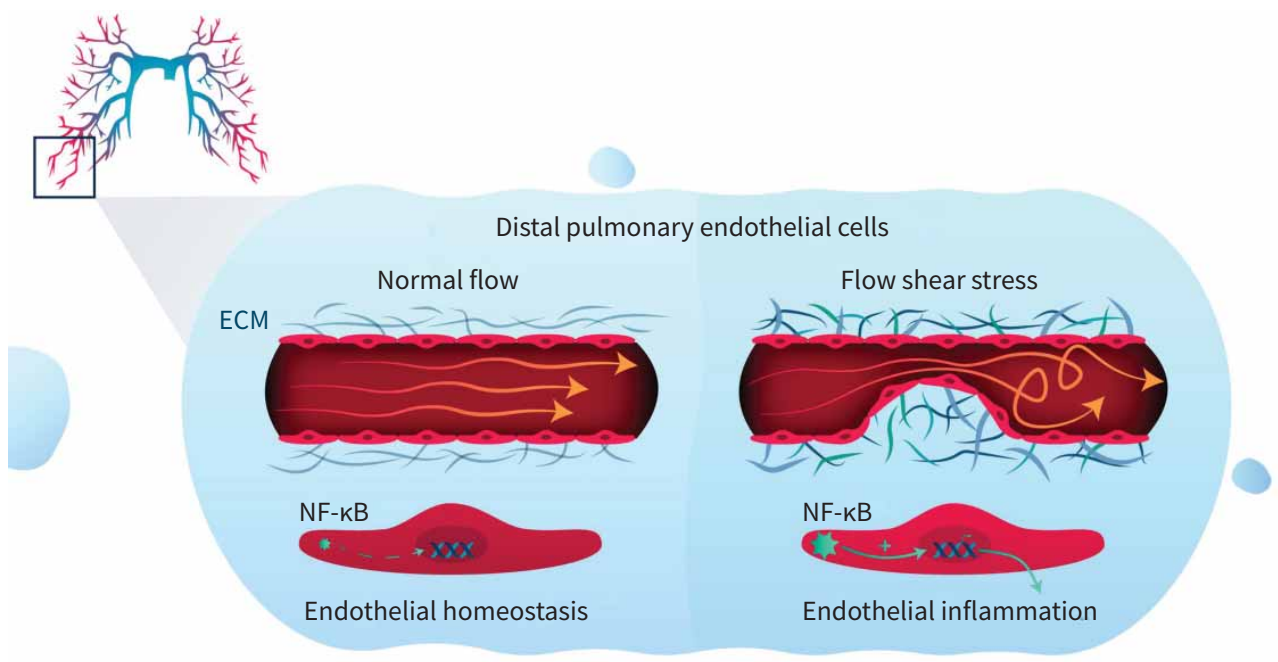

FIGURE 4 Structural alterations as a cause of inflammation. Disturbed blood flow and stiffness-induced inflammation. ECM: extra-cellular matrix. 
The smouldering fire hypothesis

We first consider the smouldering fire hypothesis. Increased vessel wall stiffness through the deposition of extra-cellular matrix, from whatever aetiology, leads to a marked pulsatile flow (with higher pressure amplitudes) that will provoke a pro-inflammatory response within the directly exposed vascular compartment, the lumen-facing endothelial cell layer of small muscular-type arteries and arterioles that lack greater amounts of elastic (amplitude-soothing) fibres. In addition, increased shear stress (due again to higher pressure amplitudes) will activate $\mathrm{NF}-\kappa \mathrm{B}$, a transcription factor that is essential for most immune/ inflammatory and cell stress responses, and contribute to the pro-inflammatory state of endothelial cells during $\mathrm{PAH}$ (figure 4) [7, 8].

In addition, the mere hypoxic state that is associated with so many respiratory and cardiovascular diseases leads to inflammatory responses by itself: at altitude, for example, hypoxia increases the levels of circulating interleukin (IL)-6 and C-reactive protein, even in healthy humans [9]. Hypoxia induces inflammation within the pulmonary artery through local expression of pro-contractile and proliferative growth factors and pro-inflammatory mediators like vascular endothelial growth factor, brain-derived neurotrophic factor or thymic stromal lymphopoietin that are generated in resident cells of the vessel wall [10]. Moreover, hypoxia induces the auto-oxidation of haemoglobin within red blood cells, and thereby increases superoxide production: higher amounts of $\mathrm{H}_{2} \mathrm{O}_{2}$ are spilled into the pulmonary circulation, and oxidative stress at the level of microvascular endothelial cells triggers the activation of the NF- $\mathrm{B}$ pathway and local recruitment of leukocytes (figure 5) [11].

However, there are other, less obvious players that might be involved in maintaining the low-level smouldering fire of inflammation within and around the pulmonary micro-vasculature. The intestinal microbiome is such a potential factor: enteric congestion due to right ventricular decompensation may lead to a dysfunctional intestinal barrier with eventual permeation of bacterial pro-inflammatory components like lipopolysaccharide into the systemic circulation [12]. These so-called pathogen-associated molecular patterns (PAMPs) induce inflammation and lead to cellular damage and stress in resident host cells; for example, in pulmonary endothelial cells that will, in turn, generate so-called damage-associated molecular patterns (DAMPs) that are part of the sterile inflammatory response. Both PAMPs and DAMPs bind to pattern-recognition receptors like Toll-like receptors or cytoplasmic NOD-like receptors and invoke strong

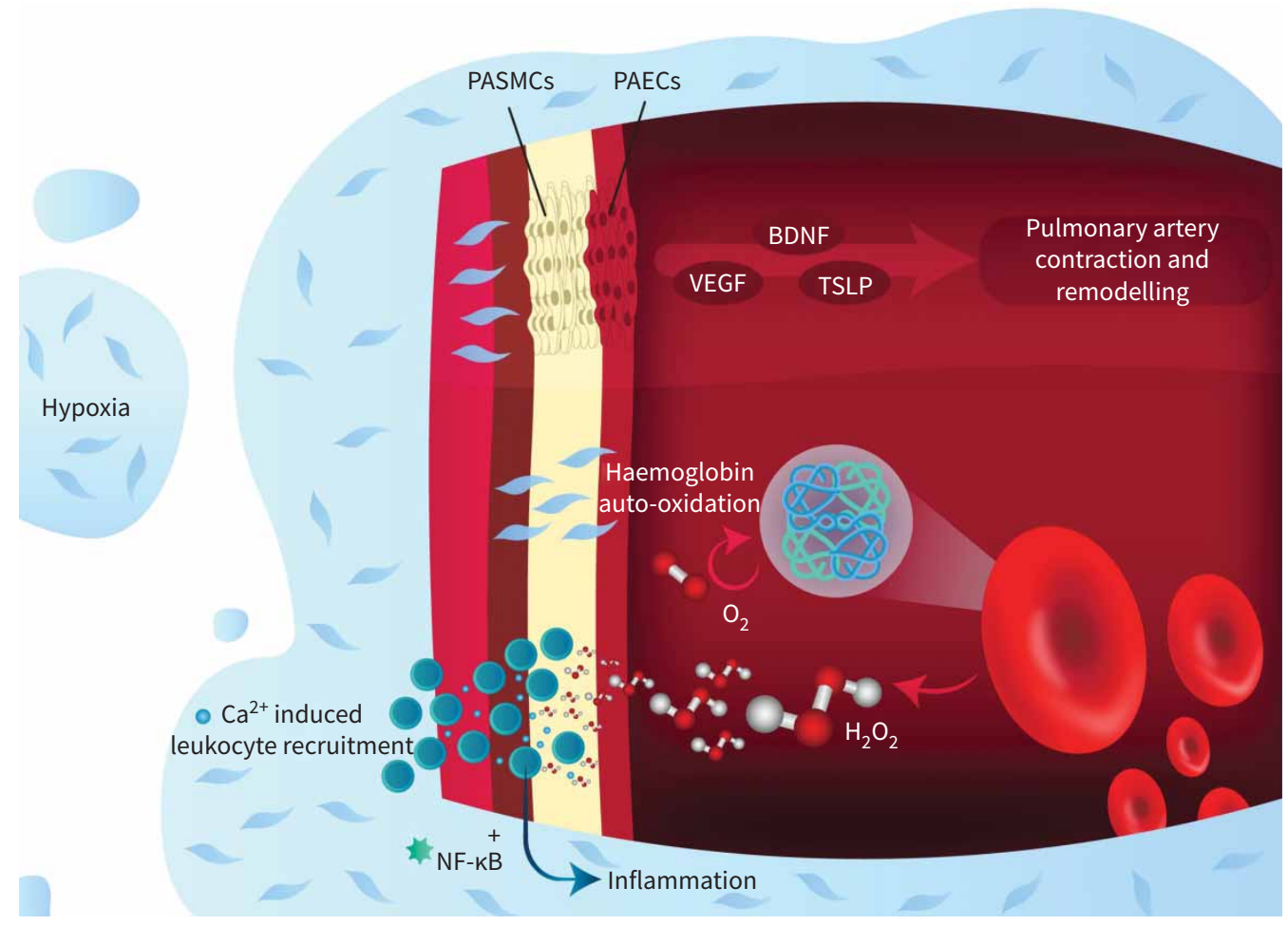

FIGURE 5 Hypoxia-induced inflammation. BDNF: brain-derived neurotrophic factor; PAEC: pulmonary arterial endothelial cell; PASMC: pulmonary arterial smooth muscle cell; TSLP: thymic stromal lymphopoietin; VEGF: vascular endothelial growth factor. 


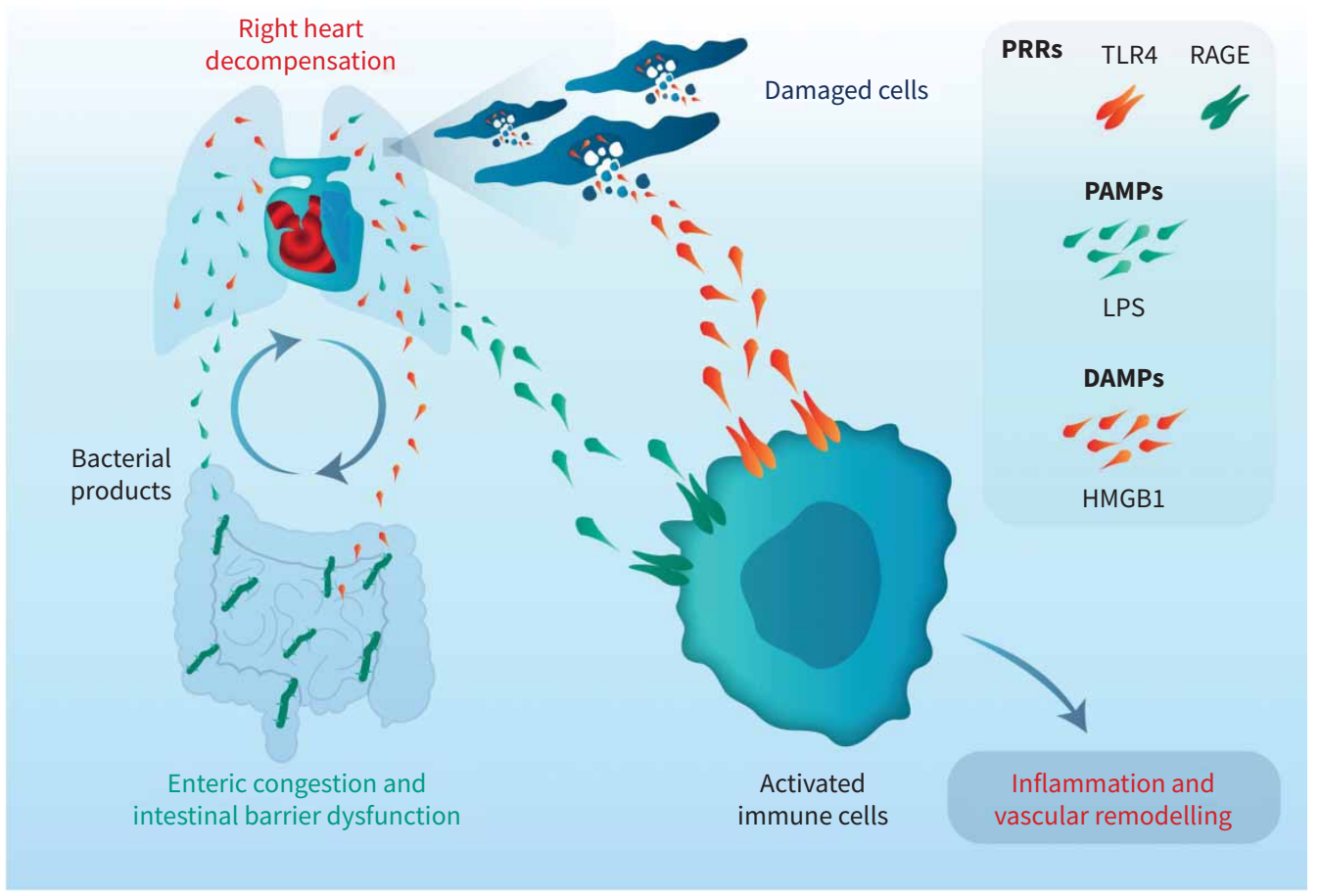

FIGURE 6 Pattern recognition receptor (PRR)-induced inflammation in pulmonary arterial hypertension. DAMP: damage-associated molecular pattern; HMGB1: high mobility group box-1; LPS: lipopolysaccharide; PAMP: pathogen-associated molecular pattern; RAGE: receptor for advanced glycation end products; TLR4: toll-like receptor 4.

local reactions with leukocytic influx, more inflammation and eventually reactive vascular remodelling [13] (figure 6). Besides pro-inflammatory properties, the commensal human microbiome could also directly influence the pulmonary vasculature [14]. Significant taxonomic and functional changes in microbial communities in the PAH cohort have been observed and some of the previously identified potential biomarkers of PAH could be derived from altered bacterial functions in PAH patients [15].

The prominent role of transcription factors with regard to inflammation and vascular remodelling has been reported and discussed in the past years. Numerous studies suggest that ETS-related gene (ERG) and Friend leukaemia integration 1 transcription factor are involved in endothelial function, proliferation, homeostasis, cellular interaction, angiogenesis, fibrosis and vascular inflammation through the gene clusters they control [16]. In experimental models with ERG-knockout, most mice die in utero, but a small fraction of survivors develops pulmonary veno-occlusive disease (PVOD), spontaneously. It has been shown that ERG is strongly diminished in human PVOD [17]. In addition, PVOD-like disease that can be induced in rats with mitomycin treatment is concomitant with loss of ERG expression, and this condition combines vascular occlusive remodelling with perivascular eosinophilic inflammation [18]. Other transcription factors that have been associated with the development of PAH-specific vascular lesions and inflammation are FoxO, hypoxia-inducible factors and twist basic helix-loop-helix transcription factor 1 (figure 7) [19].

\section{The conflagration hypothesis}

Let us have a look at what arguments could be in favour of a primary inflammatory process that initiates the pathophysiology of $\mathrm{PAH}$; in other words, the conflagration hypothesis (figure 8). Immune self-tolerance is organised and controlled by a specialised subpopulation of T-lymphocytes that are known as regulatory T-cells (Tregs). Tregs attenuate the misled immune auto-reactivity that, to a certain extent, permanently occurs, even in the healthy [20]. When the Treg-dependent immune balance is functional, autoimmune disease is avoided [21]. It has been reported that the number and function of Tregs is substantially altered in PAH [22]. In experimental models, Treg deficiency in athymic SU5416- or chronic hypoxia-treated rats leads to a severe form of $\mathrm{PH}$ that is more accentuated in female animals. Treg reconstitution in those animals coherently protects against the disease [23, 24]. Moreover, it has been shown that Treg signalling through leptin, a pleotropic hormone involved in physiological immune 

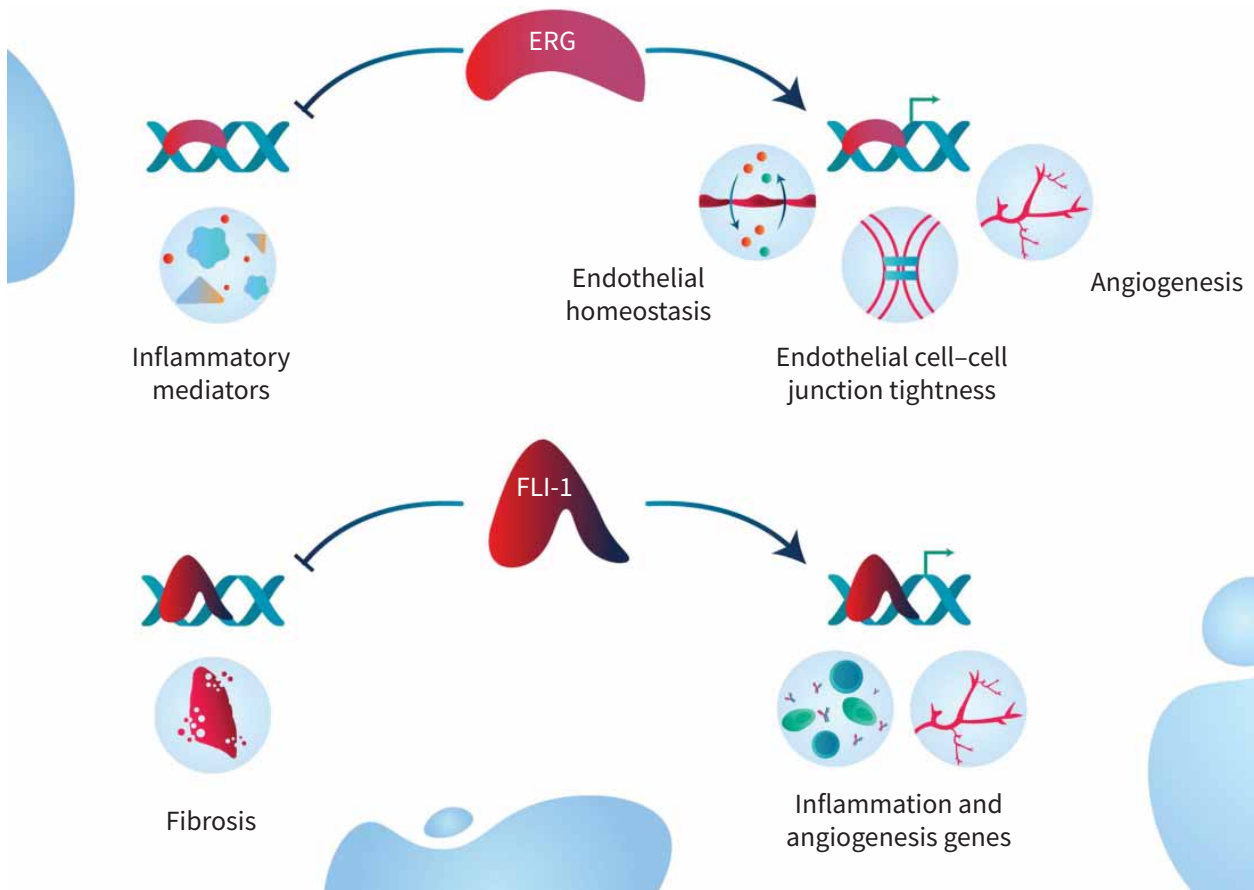

FIGURE 7 Transcription factors linking vascular remodelling and inflammation. ERG: ETS-related gene; FLI-1: Friend leukaemia integration 1 transcription factor.

\section{Autoimmunity in PAH}

Treg deregulations

Functional Treg

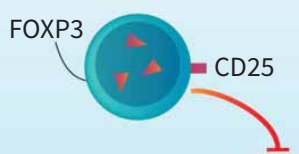

$r<+$

$t_{2}{ }^{2}$

Autoimmunity and vascular injury

Lymphoid neogenesis and auto-antibodies
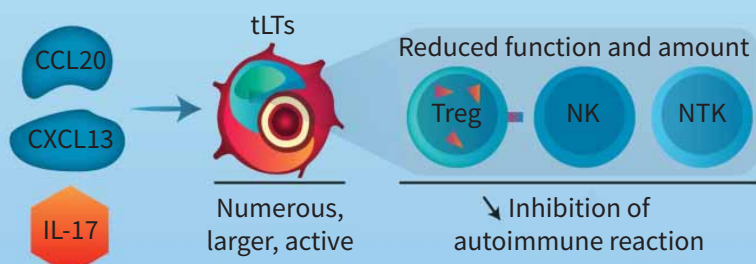

$\checkmark$ Inhibition of autoimmune reaction
Dentritic cells functional alteration

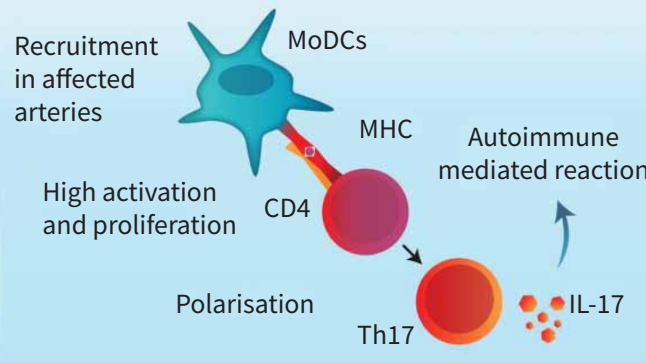

Pathogenic targets under investigation

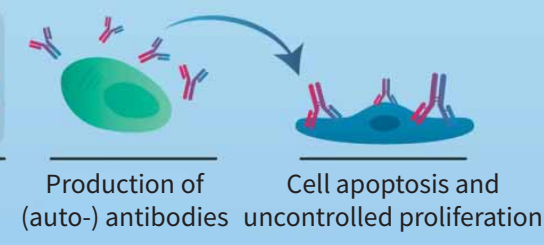

FIGURE 8 Inflammation as a cause of structural alterations: autoimmunity in pulmonary arterial hypertension (PAH). CCL20: chemokine (C-C motif) ligand 20; CXCL13: chemokine ligand 13; FOXP: Forkhead box P; IL-17: interleukin-17; MHC: major histocompatibility complex; MoDC: monocyte-derived dendritic cell; NK: natural killer cell; NKT: natural killer T-cell; Th17: T-helper 17; tLT: tertiary lymphoid tissue. 
modulation, is hampered in patients with idiopathic PAH (IPAH), as well as in patients with CTD-associated PAH and in non-PAH patients with CTD in a similar fashion, connecting the dots from PAH to mere autoimmune disease (figure 8) [25].

The link between innate and adaptive immunity is physiologically secured by dendritic cells (DCs) [26]. In the past, our group has identified and reported intense DC recruitment into the vessel wall of pulmonary arteries from patients with PAH and in PAH animal models [27-29]. We have shown that, in PAH, DCs trigger activation and proliferation of $\mathrm{CD}^{+}$T-cells, in concomitance with a T-helper 17 (Th17) immune polarisation [30] that otherwise plays a central role in the pathogenesis of multiple inflammatory autoimmune diseases, including rheumatoid arthritis (RA), multiple sclerosis, psoriasis and lupus erythematosus [31].

The conflagration concept of autoimmunity in PAH is further supported by the phenomenon of lymphoid neogenesis in lungs from patients with $\mathrm{PAH}$ : lymphocytic follicles which can be observed in the vicinity of diseased pulmonary arteries are part of so-called tertiary lymphoid tissues (tLTs) [32] that can emerge in many organs and tissues in response to persistent autoimmune-triggered inflammation and that go along with progressive organ dysfunction and degeneration, as it can be seen in RA [33]. Of note, one classic hallmark in RA histopathology of the lung is tertiary lymphocytic follicles in association with fibrotic non-specific interstitial pneumonia (NSIP)- or usual interstitial pneumonia (UIP)/idiopathic pulmonary fibrosis (IPF)-like changes of the lung parenchyma [34]. In contrast, NSIP and especially UIP virtually always present with pulmonary arterial and venous remodelling [35]. The constitution of tLT depends on chemokines, such as chemokine (C-C motif) ligand 20 and chemokine ligand 13 that have been shown to be overexpressed in explanted lungs of PAH patients [32]. IL-17, another important cytokine with regard to the constitution of tLTs and associated with human and experimental $\mathrm{PH}$, drives the formation of tertiary lymphoid follicles in lung tissue as a pillar of local antimicrobial protection, yet also enhances local inflammatory responses and autoimmune disease [30, 36, 37]. The formation of tLTs could also be favoured by the functional and numeral loss of lymphocytic populations, such as Tregs and natural killer cells (natural killer cells and natural killer T-cells) [22, 25, 38, 39].

Importantly, it has been shown that tLTs produce auto-antibodies that may be directed against the endothelial cells of pulmonary vessels, and that this aggression induces apoptosis and, eventually, critical reactive hyperproliferation of surviving endothelial cells [40]. Although auto-antibodies against smooth muscle cells and fibroblasts have been reported in PAH [41], no major common auto-antigen has been clearly associated to the disease (figure 8).

Targeting inflammation and and dysregulated immunity in PAH

From these points of view, inflammation and dysregulated immunity play a significant role in a spectrum of causes of PAH. From the perspective of identifying pathways that are targetable, IL-6 has emerged as a strong candidate. Lung-specific IL-6 overexpression transgenic mice develop PH with occlusive neointimal angioproliferative lesions that worsen with hypoxia and are composed of endothelial cells and T-lymphocytes [42]. IL-21, a Th17 cytokine, has been identified as a downstream target of IL-6 signalling in PAH. IL-6 blockade by the monoclonal anti-IL-6 receptor antibody, MR16-1, ameliorated hypoxia-induced $\mathrm{PH}$ and prevented the hypoxia-induced accumulation of Th17 cells and M2 macrophages in the lungs [43]. In a rat model of heritable PAH linked to BMPR2 mutation, pulmonary IL-6 overexpression discriminated rats that developed spontaneous PAH from rats that did not develop the condition [44]. In humans, plasma IL-6 provides incremental prognostic information in PAH, especially in patients with low brain natriuretic peptide levels [45] and explanted lungs from PAH patients display ectopic upregulation of membrane-bound IL-6 receptor on pulmonary artery smooth muscle cells [46]. However, therapeutic blockade of IL-6 signalling with tocilizumab demonstrated no significant effects on haemodynamics or exploratory secondary endpoints in heritable or IPAH. A potential improvement was noted in the small subgroup of patients with CTD-associated PAH. This raises the possibility that disease is driven by inflammation in only a subset of patients [47]. Other cytokines represent relevant therapeutic targets like tumour necrosis factor (TNF). Transgenic mice expressing chronically low levels of human TNF (TNF-Tg mice; line 3647) mimic human CTD-PAH, in which patient lungs demonstrate increased TNF signalling and significant similarities in genomic pathway dysregulation [48]. Treatment with TNF- $\alpha$ blocker etanercept (receptor Fc-based cytokine blocker) prevented and reversed monocrotaline-induced PAH in rats by reducing inflammatory cell infiltration [49]. Lastly, IL-4-/IL-13-deficient mice do not develop Schistosomiasis-associated PH, suggesting that Th2 cytokine production by $\mathrm{CD}^{+} \mathrm{T}$-cells may be required to drive $\mathrm{PH}$ in this setting [50].

In a proof-of-concept, randomised, placebo-controlled study of B-cell depletion, rituximab treatment demonstrated an acceptable safety and tolerability profile with stable systemic sclerosis PAH [51]. In the primary analysis that includes data through week 24 , the observed improvement in 6-min walking distance 


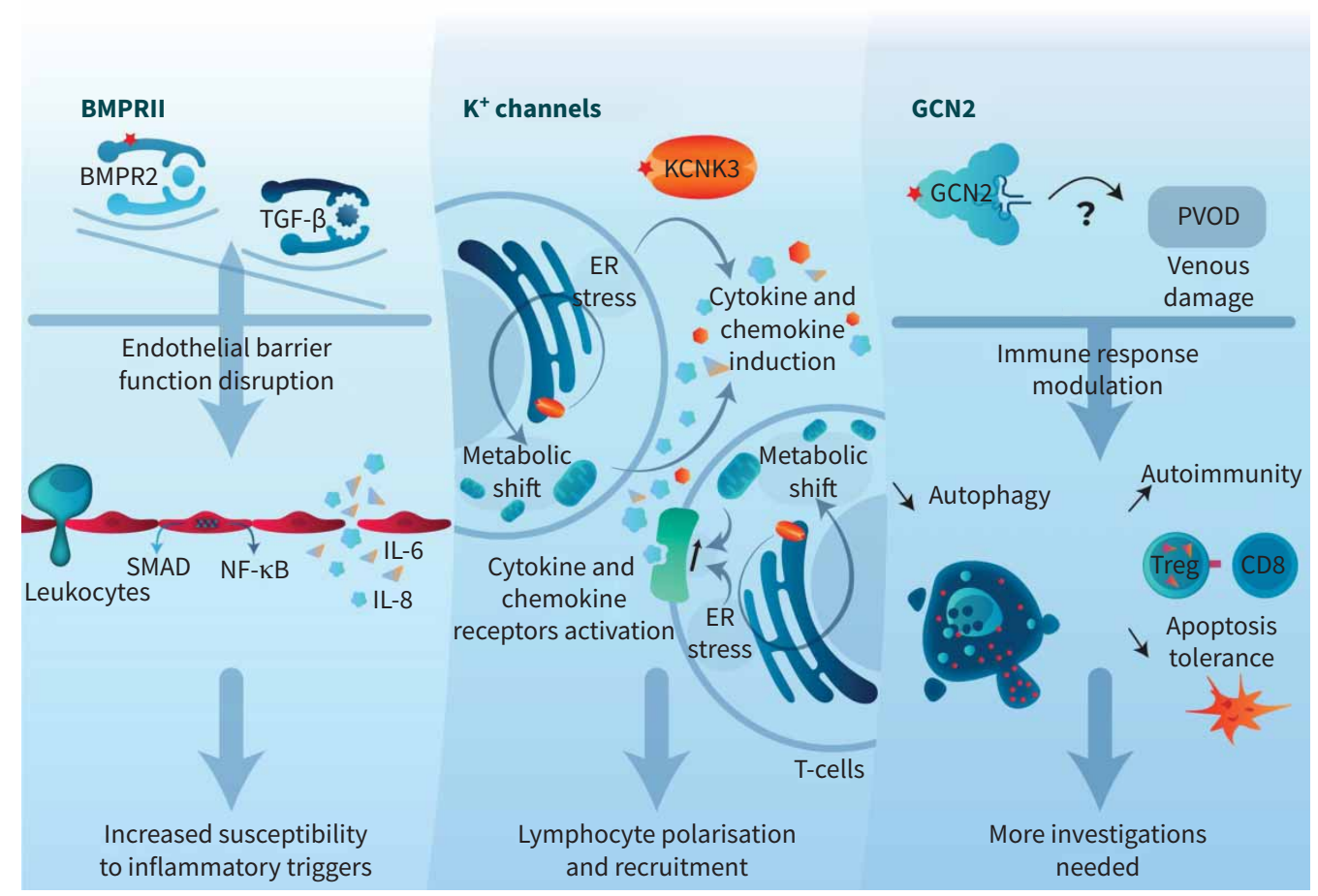

FIGURE 9 Heritable pulmonary arterial hypertension: predisposing mutations involved in immune regulation. BMPR2: bone morphogenetic protein receptor type 2; ER: endoplasmic reticulum; GCN2: general control nonderepressible 2; IL: interleukin; KCNK3: potassium two pore domain channel subfamily K member 3; PVOD: pulmonary veno-occlusive disease; TGF- $\beta$ : transforming growth factor- $\beta$.

favoured rituximab-treated patients, but this difference did not reach statistical significance. Interestingly, in a post hoc analysis, the use of machine learning allowed the identification of a panel of cytokines (low levels of rheumatoid factor, IL-2 and IL-17) that predicted a response to rituximab.

With regard to the genetic predisposition to $\mathrm{PAH}$, an increasing number of genes has been linked to $\mathrm{PAH}$ pathophysiology. The most frequently encountered mutations concern the gene BMPR2 for hereditary PAH and an important fraction of IPAH, and the gene EIF2AK4 for PAH subgroup 1.6, a particular form of $\mathrm{PAH}$ with predominating veno-occlusive remodelling and capillary proliferation (PVOD) [52]. The dysfunction of transmembrane receptor bone morphogenetic protein receptor type 2 (BMPR2) diminishes the endothelial barrier function and increases the permeability towards circulating inflammatory mediators, such as cytokines or chemokines that build a chemoattractant gradient, along which different types of leukocytes can migrate into the vessel wall of pulmonary arteries and veins (figure 9) [53, 54].

The identification of loss-of-function mutations in KCNK3 (TASK-1) [55] and ABCC8 (SUR1) [56], or gain-of-function mutations in ABCC9 (SUR2) [57], as well as polymorphisms in KCNA5 (Kv1.5) [58], which encode two potassium $\left(\mathrm{K}^{+}\right)$channels and two $\mathrm{K}^{+}$channel regulatory subunits, has revived the interest in $\mathrm{K}^{+}$channels in the pathobiology of PAH [59]. Indeed, potassium and calcium channels are involved in the regulation of pulmonary vascular tone [60]. The opening of plasma membrane $\mathrm{K}^{+}$channels leads to hyperpolarisation of pulmonary artery smooth muscle cells resulting in the closure of voltage-sensitive $\mathrm{Ca}^{2+}$ channels and subsequent vasodilation, while acute contraction of pulmonary artery smooth muscle cells is activated in part by plasma membrane $\mathrm{K}^{+}$channel inhibition-induced membrane depolarisation and subsequent $\mathrm{Ca}^{2+}$ entry through L-type $\mathrm{Ca}^{+}$channels. $\mathrm{K}^{+}$channels are also strongly involved in the proliferation of leukocytes $[61,62]$. The importance of $\mathrm{K}^{+}$channels is especially critical in T-cell activation and cytokine release. Their opening leads to plasma membrane hyperpolarisation, which in turn increases the driving force for $\mathrm{Ca}^{2+}$ influx shared by $\mathrm{Ca}^{2+}$ release-activated $\mathrm{Ca}^{2+}$ channels [63].

Potassium two pore domain channel subfamily K member 3 (KCNK3) dysfunction contributes to the development of PAH and experimental PH [64-66]. Moreover, KCNK3-mutated rats are predisposed to develop PH [67]. Paradoxically, PH spontaneously develops only slowly over 12 months in these rats, and 
while loss of KCNK3 is associated with impaired effector function in T-cells and protection against autoimmune disorders [68], KCNK3-mutated rats and mice and human PAH patients with KCNK3 mutation show increased cytokine induction and chronic immune cell activation (evidence of T-cell exhaustion) [64, 67, 69]. The question arises of how KCNK3 causes these defects. One possibility is that KCNK3 is most important not at the cell membrane, but at the membrane of the endoplasmic reticulum (ER), where it has been reported to regulate ER calcium homeostasis [70]. This explanation, recently investigated by WeST et al. [69], supposes that KCNK3 mutation primarily impacts ER calcium handling, resulting in ER stress and attendant metabolic shift, ending sequentially in cytokine/chemokine induction, cytokine/chemokine receptors activation and eventually in T-lymphocyte polarisation and recruitment (and other inflammatory cell types like macrophages) (figure 9).

General control nonderepressible 2 (GCN2), the protein product of EIF2AK4, is part of the integrated stress response and supports cell adaptation during restricted amino acid supply [71]. In myeloid cells, GCN2 is activated by catalytic degradation of tryptophan by indolamine-2,3-dioxygenase (IDO) [72]. The activation of the IDO pathway is induced by interferons during inflammation, coming from DCs, monocytes and macrophages. In addition, activation of the IDO pathway generates metabolites with immunosuppressive proprieties, such as the kynurenins [73]. In order to adapt to restricted amino acid conditions, GCN2 can activate autophagy [74]. However, the latter phenomenon opposes the inflammatory signals of macrophages during the immune response that are initiated by mitochondrial stress and the expression of cytokines such as IL-1 $\beta$ and IL-17 [75]. Hence, GCN2 appears to display a negative feedback control with regard to inflammation and its loss diminishes tolerance with regard to apoptotic cells and cellular debris and thereby shifts the balance towards autoimmunity, at least in animal models. GCN2-deficient $\mathrm{CD}^{+}$T-cells have T-cell-intrinsic proliferative and trafficking defects not observed in $\mathrm{CD}^{+}$T-cells [76]. Thus, GCN2 is also required for normal cytotoxic T-cell function, and this could explain the alterations of circulating cytotoxic cell subpopulations described in PVOD [38]. Its role in the development of human PVOD, however, remains less clearly defined (figure 9) [66].

Here are some additional thoughts on a possible inflammatory background in PAH development that underscore the difficulty of translating this concept into efficient therapeutic targets (figure 10): When analysing the explanted lungs of PAH patients who underwent year-long treatment with modern-era PAH specific therapy, we observe unresolved, stable-appearing collagen-rich obstructive vascular lesions, as well as a constant moderate chronic inflammatory infiltrate in the vessel's perimeter [77]. This may be

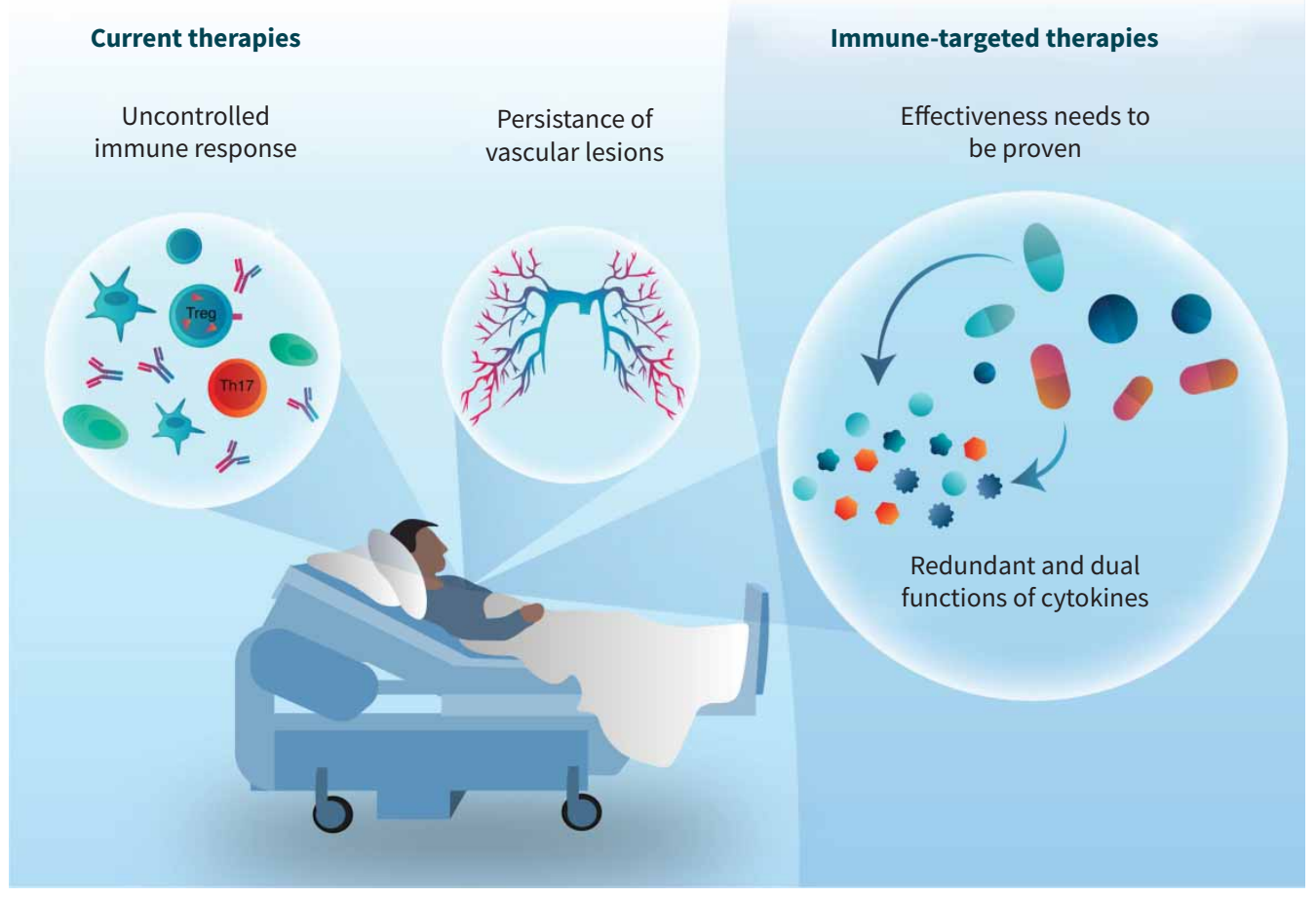

FIGURE 10 Controversies and consequences of pulmonary arterial hypertension therapy. 


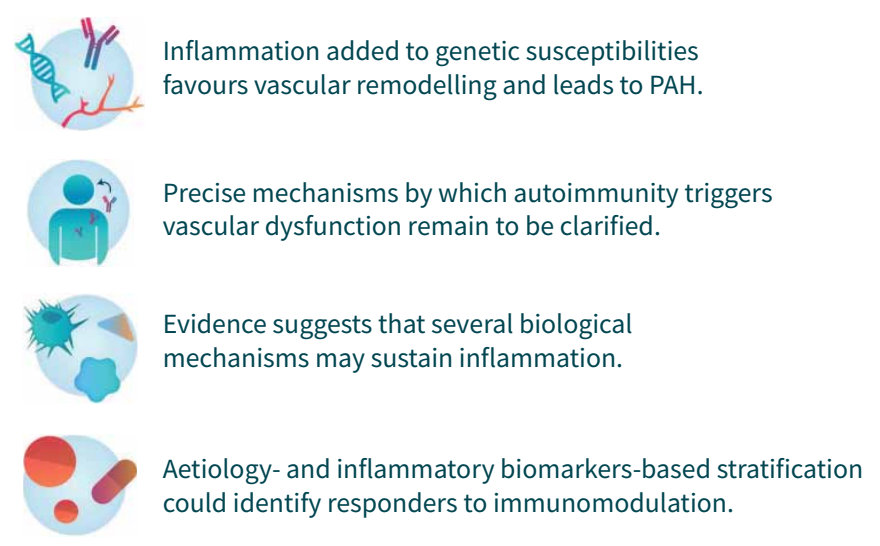

FIGURE 11 Key points to take away.

surprising to some point, since modern PAH-specific therapy has been at least experimentally shown to combine vasodilating and antiproliferative properties with immune-modulatory qualities that may attenuate exuberant inflammation [4]. Loss of immunoregulation in PAH appears to favour an ongoing chronic inflammatory process, even in end-stage PAH at a lower level [30]. It would be of particular interest to study the possible mechanisms of resistance with regard to immune modulation in patients with PAH in order to establish more personalised and hence efficient immunotherapies.

It is important to state and to admit that blocking one specific pro-inflammatory cytokine will hardly change the patient's fate or influence outcome, despite the observed and reported association of high overall cytokine levels and mortality [78]. In fact, during PAH, a vast inflammatory cytokine armada appears to be involved: therapeutically targeting only one single cytokine might be ineffective due to an important biologic redundancy of active immune mediators [79]. The dual roles of cytokines are another level of complexity. For instance, human-induced pluripotent stem cells with KCNK3 mutation and the lungs from KCNK3-mutated mice both expressed lower levels of IL-6 [69], which might be similar to the fact that IL6 maintains a stoichiometric balance in shifting signals from an active immune response to a suppressive state [80]. One should also note that IL-6 signalling prevents neointimal remodelling in Schistosomiasis-associated PH [81], possibly highlighting a dual role for IL-6. Of relevance, Sotatercept, a ligand trap with high selectivity for multiple members of the TGF- $\beta$ superfamily, significantly improved pulmonary-vascular, cardiovascular and exercise-related outcomes in PAH patients [82]. It is believed to act as a reverse-remodelling agent designed to rebalance bone morphogenetic protein/activin signalling. However, it is well known that IL-6 promotes the differentiation of Th17 cells from naïve T-cells, presumably in cooperation with TGF- $\beta$ in the lung [43]. It is possible that Sotatercept may work in other ways than those initially expected and, thus, targets some crucial cytokine/cytokine networks.

\section{Conclusion}

In conclusion, different pro-inflammatory and dysimmune triggers and pathways may - in sensible/ vulnerable patients, preferably with a genetic predisposition [83] - ignite and fuel persistent pulmonary vascular inflammation and eventually irreversible obstructive remodelling of the vessel wall. The precise mechanisms through which autoimmunity triggers vascular dysfunction are yet to be clarified.

As we have seen, there are numerous pro-inflammatory pathways and pathomechanisms, including perturbated blood-flow, vessel wall stiffness, hypoxia, bacterial translocation with pattern recognition receptor-induced inflammation and the influence of various transcription factors. Finally, up to now, immune-modulating therapeutic approaches, in general, show only very limited efficacy in PAH patients with regard to haemodynamic and clinical parameters.

We have learned from the rituximab and the tocilizumab trials that there could also be a potential benefit of addressing cells over soluble factors and that future stratified medicine approaches based on underlying (autoimmune) aetiology and inflammatory biomarkers may identify responder populations to immunomodulation. Like in other fields of applied biomedical research, for instance in novel personalised cancer therapies, these observations should not dishearten researchers within the $\mathrm{PH}$ community, but rather be understood as a challenge to pioneer a promising therapeutic landscape, almost in the range of our fingertips (figure 11). 
Provenance: Commissioned article, peer reviewed.

Acknowledgements: We thank Veronika Vikova (Biopixia, www.biopixia.com) for designing and creating the illustrations in this work.

Conflict of interest: F. Perros has nothing to disclose. M. Humbert reports grants or contracts outside the submitted work from Acceleron, Janssen, and Merck. Consulting fees, outside the submitted work received from Acceleron, Janssen, and Merck. Payment or honoraria received from AOP, Janssen, and Merck outside the submitted work for speakers bureaus. Participation on an Advisory Board for Acceleron, Janssen, and Merck, outside the submitted work. P. Dorfmüller reports receiving consulting fees from TRiCares and Sofinnova partners, outside the submitted work. Payment or honoraria for speakers bureaus from Actelion and MSD, outside the submitted work.

\section{References}

1 Simonneau G, Montani D, Celermajer DS, et al. Haemodynamic definitions and updated clinical classification of pulmonary hypertension. Eur Respir J 2019; 53, 1801913.

2 McLaughlin VV, Shah SJ, Souza R, et al. Management of pulmonary arterial hypertension. J Am Coll Cardiol 2015; 65: 1976-1997.

3 Huertas A, Perros F, Tu L, et al. Immune dysregulation and endothelial dysfunction in pulmonary arterial hypertension: a complex interplay. Circulation 2014; 129: 1332-1340.

4 Perros F, Cohen-Kaminsky S, Dorfmüller $\mathrm{P}$, et al. Inflammation in pulmonary arterial hypertension. In: Abraham D, Handler C, Dashwood M, Coghlan G, eds. Translational Vascular Medicine. London, Springer, 2012; pp. 213-229.

5 Perros F, Soubrier F, Antigny F, et al. Immunology in pulmonary vascular disorders: focus on pulmonary arterial hypertension. In: Carbone RG, Puppo F, Tapson VF, eds. Pulmonary Vascular Disorders. New York, Nova Science Publishers, 2021.

6 Cohen-Kaminsky S, Hautefort A, Price L, et al. Inflammation in pulmonary hypertension: what we know and what we could logically and safely target first. Drug Discov Today 2014; 19: 1251-1256.

7 Li M, Tan Y, Stenmark KR, et al. High pulsatility flow induces acute endothelial inflammation through overpolarising cells to activate NF-KB. Cardiovasc Eng Technol 2013; 4: 26-38.

8 Chatterjee S. Endothelial mechanotransduction, redox signaling and the regulation of vascular inflammatory pathways. Front Physiol 2018; 9: 524.

9 Hartmann G, Tschöp M, Fischer R, et al. High altitude increases circulating interleukin-6, interleukin-1 receptor antagonist and C-reactive protein. Cytokine 2000; 12: 246-252.

10 Thompson M, Britt RD, Pabelick CM, et al. Hypoxia and local inflammation in pulmonary artery structure and function. Adv Exp Med Biol 2017; 967: 325-334.

11 Huertas A, Das SR, Emin M, et al. Erythrocytes induce proinflammatory endothelial activation in hypoxia. Am J Respir Cell Mol Biol 2013; 48: 78-86.

12 Ranchoux B, Bigorgne A, Hautefort A, et al. Gut-lung connection in pulmonary arterial hypertension. Am J Respir Cell Mol Biol 2017; 56: 402-405.

13 Amarante-Mendes GP, Adjemian S, Branco LM, et al. Pattern recognition receptors and the host cell death molecular machinery. Front Immunol 2018; 9: 2379.

14 Nickel NP, Yuan K, Dorfmuller P, et al. Beyond the lungs: systemic manifestations of pulmonary arterial hypertension. Am J Respir Crit Care Med 2020; 201: 148-157.

15 Kim S, Rigatto K, Gazzana MB, et al. Altered gut microbiome profile in patients with pulmonary arterial hypertension. Hypertens 2020; 75: 1063-1071.

16 Thistlethwaite PA. Linking vascular remodeling and inflammation in pulmonary arterial hypertension: is there a common root cause? Am J Respir Cell Mol Biol 2017; 57: 15-17.

17 Lathen C, Zhang Y, Chow J, et al. ERG-APLNR axis controls pulmonary venule endothelial proliferation in pulmonary veno-occlusive disease. Circulation 2014; 130: 1179-1191.

18 Perros F, Günther S, Ranchoux B, et al. Mitomycin-induced pulmonary veno-occlusive disease: evidence from human disease and animal models. Circulation 2015; 132: 834-847.

19 Pullamsetti SS, Perros F, Chelladurai P, et al. Transcription factors, transcriptional coregulators, and epigenetic modulation in the control of pulmonary vascular cell phenotype: therapeutic implications for pulmonary hypertension (2015 Grover Conference series). Pulm Circ 2016; 6: 448-464.

20 Sakaguchi S. Naturally arising CD4+ regulatory t cells for immunologic self-tolerance and negative control of immune responses. Annu Rev Immunol 2004; 22: 531-562.

21 Wing K, Sakaguchi S. Regulatory T cells exert checks and balances on self tolerance and autoimmunity. Nat Immunol 2010; 11: 7-13.

22 Perros F, Cohen-Kaminsky S, Humbert M. Understanding the role of CD4+CD25(high) (so-called regulatory) $T$ cells in idiopathic pulmonary arterial hypertension. Respiration 2008; 75: 253-256. 
23 Tamosiuniene R, Tian W, Dhillon G, et al. Regulatory T cells limit vascular endothelial injury and prevent pulmonary hypertension. Circ Res 2011; 109: 867-879.

24 Tamosiuniene R, Manouvakhova $\mathrm{O}$, Mesange $\mathrm{P}$, et al. Dominant role for regulatory $\mathrm{T}$ cells in protecting females against pulmonary hypertension. Circ Res 2018; 122: 1689-1702.

25 Huertas A, Tu L, Gambaryan N, et al. Leptin and regulatory T-lymphocytes in idiopathic pulmonary arterial hypertension. Eur Respir J 2012; 40: 895-904.

26 Lambrecht BN, Hammad $\mathrm{H}$. The role of dendritic and epithelial cells as master regulators of allergic airway inflammation. Lancet 2010; 376: 835-843.

27 Perros F, Dorfmüller $\mathrm{P}$, Souza $\mathrm{R}$, et al. Dendritic cell recruitment in lesions of human and experimental pulmonary hypertension. Eur Respir J 2007; 29: 462-468.

28 Dorfmüller $\mathrm{P}$, Chaumais M-C, Giannakouli M, et al. Increased oxidative stress and severe arterial remodeling induced by permanent high-flow challenge in experimental pulmonary hypertension. Respir Res 2011; 12: 119 .

29 van Uden D, Koudstaal T, van Hulst JAC, et al. Central role of dendritic cells in pulmonary arterial hypertension in human and mice. Int J Mol Sci 2021; 22: 1756.

30 Hautefort A, Girerd B, Montani D, et al. T-helper 17 cell polarisation in pulmonary arterial hypertension. Chest 2015; 147: 1610-1620.

31 Han L, Yang J, Wang X, et al. Th17 cells in autoimmune diseases. Front Med 2015; 9: 10-19.

32 Perros F, Dorfmüller $\mathrm{P}$, Montani $\mathrm{D}$, et al. Pulmonary lymphoid neogenesis in idiopathic pulmonary arterial hypertension. Am J Respir Crit Care Med 2012; 185: 311-321.

33 Neyt K, Perros F, GeurtsvanKessel CH, et al. Tertiary lymphoid organs in infection and autoimmunity. Trends Immunol 2012; 33: 297-305.

34 Hashisako M, Fukuoka J. Pathology of idiopathic interstitial pneumonias. Clin Med Insights Circ Respir Pulm Med 2016; 9: 123-133.

35 Hallowell RW, Reed RM, Fraig M, et al. Severe pulmonary hypertension in idiopathic nonspecific interstitial pneumonia. Pulm Circ 2012; 2: 101-106.

36 Rangel-Moreno J, Carragher DM, de la Luz Garcia-Hernandez M, et al. The development of inducible bronchus-associated lymphoid tissue depends on IL-17. Nat Immunol 2011; 12: 639-646.

37 Altmann DM. T-cell immunology of the lung: maintaining the balance between host defence and immune pathology. Immunology 2019; 156: 1-2.

38 Perros F, Cohen-Kaminsky S, Gambaryan N, et al. Cytotoxic cells and granulysin in pulmonary arterial hypertension and pulmonary veno-occlusive disease. Am J Respir Crit Care Med 2013; 187: 189-196.

39 Ormiston ML, Chang C, Long LL, et al. Impaired natural killer cell phenotype and function in idiopathic and heritable pulmonary arterial hypertension. Circulation 2012; 126: 1099-1109.

40 Colvin KL, Cripe PJ, Ivy DD, et al. Bronchus-associated lymphoid tissue in pulmonary hypertension produces pathologic autoantibodies. Am J Respir Crit Care Med 2013; 188: 1126-1136.

41 Mouthon L, Guillevin L, Humbert M. Pulmonary arterial hypertension: an autoimmune disease? Eur Respir J 2005; 26: 986-988.

42 Steiner MK, Syrkina OL, Kolliputi N, et al. Interleukin-6 overexpression induces pulmonary hypertension. Circ Res 2009; 104: 236-244.

43 Hashimoto-Kataoka T, Hosen N, Sonobe T, et al. Interleukin-6/interleukin-21 signaling axis is critical in the pathogenesis of pulmonary arterial hypertension. Proc Natl Acad Sci USA 2015; 112: E2677-E2686.

44 Hautefort A, Mendes-Ferreira P, Sabourin J, et al. Bmpr2 mutant rats develop pulmonary and cardiac characteristics of pulmonary arterial hypertension. Circulation 2019; 139: 932-948.

45 Heresi GA, Aytekin M, Hammel JP, et al. Plasma interleukin-6 adds prognostic information in pulmonary arterial hypertension. Eur Respir J 2014; 43: 912-914.

46 Tamura Y, Phan C, Tu L, et al. Ectopic upregulation of membrane-bound IL6R drives vascular remodeling in pulmonary arterial hypertension. J Clin Invest 2018; 128: 1956-1970.

47 Toshner M, Church C, Harbaum L, et al. Mendelian randomisation and experimental medicine approaches to IL-6 as a drug target in PAH. Eur Respir J 2021; in press [https://doi.org/10.1183/13993003.02463-2020].

48 Bell RD, White RJ, Garcia-Hernandez ML, et al. Tumor necrosis factor induces obliterative pulmonary vascular disease in a novel model of connective tissue disease-associated pulmonary arterial hypertension. Arthritis Rheumatol 2020; 72: 1759-1770.

49 Zhang L-L, Lu J, Li M-T, et al. Preventive and remedial application of etanercept attenuate monocrotaline-induced pulmonary arterial hypertension. Int J Rheum Dis 2016; 19: 192-198.

50 Kumar R, Mickael C, Kassa B, et al. Th2 $\mathrm{CD}^{+} \mathrm{T}$ cells are necessary and sufficient for schistosoma-pulmonary hypertension. J Am Heart Assoc 2019; 8: e013111.

51 Zamanian RT, Badesch D, Chung L, et al. Safety and efficacy of B-cell depletion with rituximab for the treatment of systemic sclerosis-associated pulmonary arterial hypertension: a multicenter, double-blind, randomised, placebo-controlled trial. Am J Respir Crit Care Med 2021; 204: 209-221. 
52 Morrell NW, Aldred MA, Chung WK, et al. Genetics and genomics of pulmonary arterial hypertension. Eur Respir J 2019; 53: 1801899.

53 Tielemans B, Delcroix M, Belge C, et al. TGF $\beta$ and BMPRIl signalling pathways in the pathogenesis of pulmonary arterial hypertension. Drug Discov Today 2019; 24: 703-716.

54 Tielemans B, Stoian L, Gijsbers R, et al. Cytokines trigger disruption of endothelium barrier function and p38 MAP kinase activation in BMPR2-silenced human lung microvascular endothelial cells. Pulm Circ 2019; 9: 2045894019883607.

55 Ma L, Roman-Campos D, Austin ED, et al. A novel channelopathy in pulmonary arterial hypertension. $N$ Engl J Med 2013; 369: 351-361.

56 Bohnen MS, Ma L, Zhu N, et al. Loss-of-function ABCC8 mutations in pulmonary arterial hypertension. Circ Genom Precis Med 2018; 11: e002087.

57 Ma A, Gurnasinghani S, Kirk EP, et al. Glibenclamide treatment in a Cantú syndrome patient with a pathogenic ABCC9 gain-of-function variant: initial experience. Am J Med Genet A 2019; 179: 1585-1590.

58 Wipff J, Dieudé $P$, Guedj $M$, et al. Association of a KCNA5 gene polymorphism with systemic sclerosis-associated pulmonary arterial hypertension in the European Caucasian population. Arthritis Rheum 2010; 62: 3093-3100.

59 Le Ribeuz H, Capuano V, Girerd B, et al. Implication of potassium channels in the pathophysiology of pulmonary arterial hypertension. Biomolecules 2020; 10: 1261.

60 Olschewski A, Veale EL, Nagy BM, et al. TASK-1 (KCNK3) channels in the lung: from cell biology to clinical implications. Eur Respir J 2017; 50: 1700754.

61 Cahalan MD, Chandy KG. Ion channels in the immune system as targets for immunosuppression. Curr Opin Biotechnol 1997; 8: 749-756.

62 Panyi G, Varga Z, Gáspár R. Ion channels and lymphocyte activation. Immunol Lett 2004; 92: 55-66.

63 Prakriya M, Lewis RS. CRAC channels: activation, permeation, and the search for a molecular identity. Cell Calcium 2003; 33: 311-321.

64 Antigny F, Hautefort A, Meloche J, et al. Potassium channel subfamily K member 3 (KCNK3) contributes to the development of pulmonary arterial hypertension. Circulation 2016; 133: 1371-1385.

65 Ranchoux B, Günther S, Quarck R, et al. Chemotherapy-induced pulmonary hypertension: role of alkylating agents. Am J Pathol 2015; 185: 356-371.

66 Manaud G, Nossent EJ, Lambert M, et al. Comparison of human and experimental pulmonary veno-occlusive disease. Am J Respir Cell Mol Biol 2020; 63: 118-131.

67 Lambert M, Capuano V, Boet A, et al. Characterisation of Kcnk3-mutated rat, a novel model of pulmonary hypertension. Circ Res 2019; 125: 678-695.

68 Bittner S, Meuth SG, Göbel K, et al. TASK1 modulates inflammation and neurodegeneration in autoimmune inflammation of the central nervous system. Brain J Neurol 2009; 132: 2501-2516.

69 West JD, Austin ED, Rizzi EM, et al. KCNK3 mutation causes altered immune function in pulmonary arterial hypertension patients and mouse models. Int J Mol Sci 2021; 22: 5014.

70 Jacobson DA, Shyng S-L. Ion channels of the islets in type 2 diabetes. J Mol Biol 2020; 432: 1326-1346.

71 Santos-Ribeiro D, Godinas L, Pilette C, et al. The integrated stress response system in cardiovascular disease. Drug Discov Today 2018; 23: 920-929.

72 Munn DH, Sharma MD, Baban B, et al. GCN2 kinase in T cells mediates proliferative arrest and anergy induction in response to indoleamine 2,3-dioxygenase. Immunity 2005; 22: 633-642.

73 Nguyen NT, Kimura A, Nakahama T, et al. Aryl hydrocarbon receptor negatively regulates dendritic cell immunogenicity via a kynurenine-dependent mechanism. Proc Natl Acad Sci USA 2010; 107: 19961-19966.

74 Ravindran R, Khan N, Nakaya HI, et al. Vaccine activation of the nutrient sensor GCN2 in dendritic cells enhances antigen presentation. Science 2014; 343: 313-317.

75 Ravindran R, Loebbermann J, Nakaya HI, et al. The amino acid sensor GCN2 controls gut inflammation by inhibiting inflammasome activation. Nature 2016; 531: 523-527.

76 Van de Velde L-A, Guo X-ZJ, Barbaric L, et al. Stress kinase GCN2 controls the proliferative fitness and trafficking of cytotoxic $T$ cells independent of environmental amino acid sensing. Cell Rep 2016; 17: 2247-2258.

77 Stacher E, Graham BB, Hunt JM, et al. Modern age pathology of pulmonary arterial hypertension. Am J Respir Crit Care Med 2012; 186: 261-272.

78 Cracowski J-L, Chabot F, Labarère J, et al. Proinflammatory cytokine levels are linked to death in pulmonary arterial hypertension. Eur Respir J 2014; 43: 915-917.

79 Nicola NA. Cytokine pleiotropy and redundancy: a view from the receptor. Stem Cells 1994; 12: Suppl. 1, 3-12.

80 Fisher DT, Appenheimer MM, Evans SS. The two faces of IL-6 in the tumor microenvironment. Semin Immunol 2014; 26: 38-47.

81 Graham BB, Chabon J, Kumar R, et al. Protective role of IL-6 in vascular remodeling in Schistosoma pulmonary hypertension. Am J Respir Cell Mol Biol 2013; 49: 951-959. 
82 Humbert M, McLaughlin V, Gibbs JSR, et al. Sotatercept for the treatment of pulmonary arterial hypertension. N Engl J Med 2021; 384: 1204-1215.

83 Tian W, Jiang X, Sung YK, et al. Phenotypically silent bone morphogenetic protein receptor 2 mutations predispose rats to inflammation-induced pulmonary arterial hypertension by enhancing the risk for neointimal transformation. Circulation 2019; 140: 1409-1425. 